\title{
Hybrid-PIC modeling of a high-voltage, high-specific-impulse Hall thruster
}

\author{
Brandon D. Smith* and Iain D. Boyd ${ }^{\dagger}$ \\ Nonequilibrium Gas and Plasma Dynamics Laboratory \\ University of Michigan, Ann Arbor, Michigan, 48109, USA \\ and \\ Hani Kamhawi ${ }^{\ddagger}$ and Wensheng Huang ${ }^{\S}$ \\ NASA Glenn Research Center, Cleveland, Ohio, 44135, USA
}

\begin{abstract}
The primary life-limiting mechanism of Hall thrusters is the sputter erosion of the discharge channel walls by high-energy propellant ions. Because of the difficulty involved in characterizing this erosion experimentally, many past efforts have focused on numerical modeling to predict erosion rates and thruster lifespan, but those analyses were limited to Hall thrusters operating in the $200-400 \mathrm{~V}$ discharge voltage range. Thrusters operating at higher discharge voltages $\left(V_{d} \geq 500 \mathrm{~V}\right)$ present an erosion environment that may differ greatly from that of the lower-voltage thrusters modeled in the past. In this work, HPHall, a well-established hybrid-PIC code, is used to simulate NASA's High-Voltage Hall Accelerator (HiVHAc) at discharge voltages of 300,400 , and $500 \mathrm{~V}$ as a first step towards modeling the discharge channel erosion. It is found that the model accurately predicts the thruster performance at all operating conditions to within $6 \%$. The model predicts a normalized plasma potential profile that is consistent between all three operating points, with the acceleration zone appearing in the same approximate location. The expected trend of increasing electron temperature with increasing discharge voltage is observed. An analysis of the discharge current oscillations shows that the model predicts oscillations that are much greater in amplitude than those measured experimentally at all operating points, suggesting that the differences in oscillation amplitude are not strongly associated with discharge voltage.
\end{abstract}

\section{Nomenclature}

$\begin{array}{llr}\alpha_{a} & \text { Bohm coefficient for region } a & \\ \delta_{j} & \text { Charge-weighted beam divergence angle } & \\ \eta_{a} & \text { Thrust-derived anode efficiency } & \mathrm{T} \cdot \mathrm{m}^{2} \\ \lambda & \text { Magnetic stream function } & \mathrm{m}^{2} / \mathrm{V} \cdot \mathrm{s} \\ \mu_{e, \perp} & \text { Electron cross-field mobility } & \mathrm{s}^{-1} \\ \nu_{e} & \text { Total effective electron collision frequency } & \mathrm{s}^{-1} \\ \nu_{b} & \text { Anomalous Bohm collision frequency } & \mathrm{s}^{-1} \\ \nu_{e i} & \text { Electron-ion collision frequency } & \mathrm{s}^{-1} \\ \nu_{e n} & \text { Electron-neutral collision frequency } & \mathrm{s}^{-1} \\ \nu_{w} & \text { Effective wall collision frequency for electrons } & \mathrm{V} \\ \Omega_{e} & \text { Electron Hall parameter } & \mathrm{V} \\ \phi, \phi^{*} & \text { Local and thermalized plasma potential } & \end{array}$

*Ph.D. Candidate, Department of Aerospace Engineering, bradenis@umich.edu, AIAA Student Member.

$\dagger$ James E. Knott Professor, Department of Aerospace Engineering, iainboyd@umich.edu, AIAA Fellow.

${ }^{\ddagger}$ Research Engineer, Propulsion and Propellants, hani.kamhawi-1@nasa.gov, AIAA Associate Fellow.

$\S$ Research Engineer, Propulsion and Propellants, wensheng.huang@nasa.gov, AIAA Member. 


$\begin{array}{llr}B & \text { Magnetic field } & \mathrm{T} \\ e & \text { Elementary charge } & \mathrm{C} \\ F_{t h} & \text { Thrust force } & \mathrm{N} \\ I_{b} & \text { Ion beam current } & \mathrm{A} \\ I_{d} & \text { Discharge current } & \mathrm{A} \\ I_{s p} & \text { Anode specific impulse } & \mathrm{s} \\ j_{z} & \text { Axial ion current density } & \mathrm{A} / \mathrm{m}^{2} \\ k_{B} & \text { Boltzmann constant } & \mathrm{J} / \mathrm{eV} \\ \dot{m}_{a} & \text { Anode mass flow rate } & \mathrm{kg} / \mathrm{s} \\ m_{e} & \text { Electron mass } & \mathrm{kg} \\ n_{e}, n_{e, 0} & \text { Local and reference electron density } & \mathrm{m}^{-3} \\ p_{c} & \text { Operating chamber pressure measured at thruster location, corrected for xenon } & \mathrm{Torr} \\ r & \text { Radial coordinate } & \mathrm{m} \\ T_{e} & \text { Electron temperature } & \mathrm{eV} \\ V_{d} & \text { Discharge voltage } & \mathrm{V} \\ z & \text { Axial coordinate } & \mathrm{m}\end{array}$

\section{Introduction}

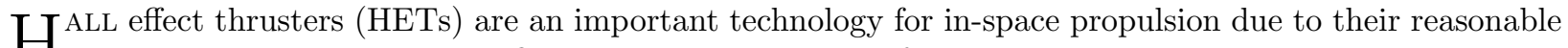

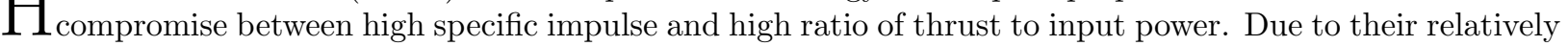
low total thrust, however, Hall thrusters must operate for 10,000 hours or more in order to achieve the total impulse necessary to complete the very missions for which these devices are ideal. The lifespan of Hall thrusters is currently limited by the erosion of the discharge channel walls via sputtering by high-energy propellant ions from the plasma. Experimental characterization of sputter erosion has proven difficult because the sputter yields caused by ions in the energy range of interest to Hall thrusters are difficult to detect with presently available measurement tools. Additionally, life-testing a thruster at high vacuum conditions is extremely time-consuming and costly. These considerations make numerical models a valuable tool with which to estimate Hall thruster lifespan.

One numerical model that has been frequently applied to Hall thrusters in the past is HPHall, a hybrid code in which electrons are modeled as a quasi-1D fluid and heavy species are modeled using a particle-in-cell (PIC) method. ${ }^{1}$ Originally developed by Fife in the 1990s, HPHall has undergone significant development in the past decade to improve its capabilities ${ }^{2-8}$ and has been used as a tool for predicting discharge channel erosion. ${ }^{2,9,10}$ However, these efforts have focused largely on the simulation of Hall thrusters operating with discharge voltages between 200-400 V. Hall thrusters operating at higher voltages present plasma conditions and erosion environments different from what has been modeled using HPHall or other codes in the past. Thus, the validity of these models at high discharge voltages has not yet been well determined.

In this work, the ability of HPHall to model NASA's High-Voltage Hall Accelerator (HiVHAc) over a range of discharge voltages from 300 to $500 \mathrm{~V}$ is evaluated. In Section II-A, an overview of HiVHAc and the experimental testing procedure is given. In Section II-B, the details of the numerical model are explained. In Section III-A, the accuracy of the model in predicting thruster performance is assessed. In Section III-B, the simulated internal plasma properties of the thruster at $500 \mathrm{~V}$ are compared to those at 300 and $400 \mathrm{~V}$ to determine whether the model predicts any changes in plasma characteristics with increasing discharge voltage. In Section III-C, the discharge current oscillations predicted by the model are compared to those measured experimentally. Finally, in section IV, the results of the current study are summarized, and the work required to meet the long-term goal of applying HPHall as an erosion prediction tool for HiVHAc is outlined.

\section{Technical approach}

\section{A. High Voltage Hall Accelerator and testing}

The High Voltage Hall Accelerator (HiVHAc) project ${ }^{11,12}$ is a Hall thruster project currently being conducted by NASA Glenn Research Center (GRC) and Aerojet. Intended for use on NASA Discovery-Class missions, the thruster's design characteristics include a wide throttling range and a novel life-extending chan- 
nel replacement mechanism. The current engineering development unit (EDU2) has demonstrated operation at discharge voltages up to $650 \mathrm{~V}$ and discharge powers in excess of $4 \mathrm{~kW} .{ }^{13}$ When operating at high voltages, the thruster is capable of generating a specific impulse of nearly $2700 \mathrm{~s}$, and when operating at low voltages, it can achieve a thrust-to-power ratio competitive with other state-of-the-art Hall thrusters.

Recent testing of the HiVHAc EDU2 took place in Vacuum Facility 5 (VF-5) at GRC in

Table 1: HiVHAc EDU2 performance as measured in VF-5 for several operating points.

\begin{tabular}{|c|c|c|c|c|}
\hline$V_{d}(\mathrm{~V})$ & $\dot{m}_{a}(\mathrm{mg} / \mathrm{s})$ & $I_{d}(\mathrm{~A})$ & $F_{t h}(\mathrm{mN})$ & $p_{c}$ (Torr) \\
\hline 300.3 & 10.21 & 9.96 & 186 & $2.7 \cdot 10^{-6}$ \\
400.8 & 8.29 & 8.00 & 173 & $2.2 \cdot 10^{-6}$ \\
500.0 & 7.13 & 6.97 & 169 & $1.7 \cdot 10^{-6}$ \\
\hline
\end{tabular}

April-May 2013. VF-5 is a $18.3 \mathrm{~m}$ long, $4.6 \mathrm{~m}$

diameter cylindrical vacuum chamber with cryogenic and oil diffusion pumping systems capable of maintaining a no-load background pressure of $1 \times 10^{-7}$ Torr. The test diagnostics suite included a Faraday probe to characterize the distribution of current in the ion beam, an oscilloscope to measure discharge current oscillations, and an inverted pendulum thrust stand. The Faraday probe was swept through a $180^{\circ}$ arc in the plume for each measurement. The thruster was tested at several operating points in order to characterize the behavior of the thruster at different conditions. Table 1 shows the thruster performance at a selection of operating conditions as measured in VF-5. For all cases, the thruster was run with xenon propellant.

\section{B. Numerical model and simulation inputs}

HPHall is a computational model for Hall thrusters that uses the hybrid-PIC method, in which ions and neutral propellant atoms are modeled using an axisymmetric particle-in-cell (PIC) approach and electrons are modeled as a quasi-1D fluid. The plasma is assumed to be quasi-neutral throughout the simulation domain, with the plasma density computed in the PIC submodel. The plasma potential is computed using the well-known thermalized potential approximation:

$$
\phi^{*}(\lambda)=\phi-\frac{k_{B} T_{e}(\lambda)}{e} \ln \left(\frac{n_{e}}{n_{e, 0}}\right)
$$

where $\phi^{*}$ and $T_{e}$ are constant along magnetic field lines. The thermalized potential $\phi^{*}$ is found by momentum conservation across magnetic field lines and the electron temperature $T_{e}$ is computed via energy conservation across field lines. The magnetic field itself is assumed to be that imposed by the magnetic circuit of the thruster and is included as one of the model's inputs. While the thermalized potential approximation simplifies the treatment of electrons in the simulations, the large difference between the characteristic time scales for the electrons and the heavy species must still be accounted for. HPHall accomplishes this by using an electron time step that is a fraction of the base ion and neutral time step, integrating the electron equations many times for each integration of the ion and neutral equations.

Since its initial development by Fife, ${ }^{1}$ HPHall has undergone several modifications to improve its capabilities. First, Gamero-Castaño and Katz implemented channel erosion and improved sheath models. ${ }^{2}$ Later, Parra et al. made enhancements to the heavy particle integration and weighting, and also to the cross-field electron transport model. ${ }^{3,4}$ Hofer et al. made additional improvements to the cross-field transport, heavy species, and erosion submodels. ${ }^{6-9}$ Finally, Huismann added a direct-simulation Monte Carlo (DSMC) collision module for handling charge- and momentum-exchange collisions between ions and neutrals ${ }^{14}$ leading to the most recent version of the code, called HPHall-3. This is the version used in the current work.

One of the principal characteristics of HPHall and other hybrid-PIC codes is their treatment of the transport of electrons across magnetic field lines. In general, the cross-field mobility of electrons can be written as

$$
\mu_{e, \perp}=\frac{e}{m_{e} \nu_{e}} \frac{1}{1+\Omega_{e}^{2}} \approx \frac{m_{e} \nu_{e}}{e B^{2}}
$$

for large $\Omega_{e}$, as is the case in Hall thrusters. The effective collision frequency can be written as

$$
\nu_{e}=\nu_{e i}+\nu_{e n}+\nu_{w}+\nu_{b} .
$$


The anomalous Bohm collision frequency $\nu_{b}$ is intended to account for the discrepancy between the classicallyderived cross-field mobility of electrons and the observed axial electron current in Hall thrusters. It is modeled in a semi-empirical manner as

$$
\nu_{b}=\alpha \frac{1}{16} \frac{e B}{m_{e}}
$$

where $\alpha$ is a user-defined coefficient. The first version of HPHall used a single value of $\alpha$ throughout the simulation domain. ${ }^{1}$ Hofer et al. later split the domain into two distinct regions, each with its own value of $\alpha,{ }^{6,7,9}$ and then into three regions ${ }^{8}$ in order to better match experimentally observed axial variations in the electron Hall parameter. These regions are denoted by $\alpha_{c}$ for the near-anode region, $\alpha_{e}$ for the thruster exit, and $\alpha_{p}$ for the plume. Since the cross-field transport of electrons has a substantial impact on thruster behavior, it is necessary for the user to adjust the values of $\alpha$ and the locations of each discharge region until adequate agreement between the model and experiment is achieved. For this work, the location of each of the three regions is tuned to roughly match the measured discharge current using values of $\alpha$ found for the $\mathrm{H} 66 \mathrm{~kW}$ Hall thruster. ${ }^{8}$ The plume coefficient $\alpha_{p}$ is then fixed to 10 and the other two values are adjusted for closer matching to the measured discharge current. While adjusting the Bohm coefficients, fine adjustment of the location of each region is allowed as necessary.

The thruster operating points modeled in this work are those shown in Table 1. The mesh used is the same for all cases and consists of $70 \times 30$ cells. The magnetic fields are generated using the commercial magnetic field solver MagNet and validated against measurements of the thruster's magnetic field taken immediately after testing in VF-5. The base ion and neutral time step is $5 \times 10^{-8} \mathrm{~s}$ and the electron time step is $1 / 1000$ the base time step. The position of the effective cathode is determined by running a complete simulation with a given cathode position, recording the thrust, moving the cathode downstream, and repeating until the average thrust becomes independent of the cathode position. Simulations are run by first processing 20,000 time steps including only neutrals, then turning on the plasma and running for 50,000 time steps. Doublycharged ions, the facility background pressure, and the charge-exchange collision algorithm are included in all cases. The wall (real) time required for one complete simulation is roughly 5 hours.

\section{Results}

\section{A. Time-averaged performance}

The computed time-averaged performance of HiVHAc EDU2 and the associated Bohm diffusion coefficients are shown in Table 2 for each of the throttling points shown in Table 1. The predicted and measured values of discharge current, ion beam current, and thrust differ by no more than $6 \%, 2 \%$, and $3 \%$ for 300,400 , and $500 \mathrm{~V}$ operation, respectively.

Table 2: HiVHAc EDU2 performance as computed by HPHall.

\begin{tabular}{|c|c|c|c|c|c|c|c|c|c|c|c|}
\hline \multirow{2}{*}{$V_{d}(\mathrm{~V})$} & \multirow{2}{*}{$\alpha_{c}$} & \multirow{2}{*}{$\alpha_{e}$} & \multirow{2}{*}{$\alpha_{p}$} & \multicolumn{2}{|c|}{$I_{d}(\mathrm{~A})$} & \multicolumn{2}{c|}{$I_{b}(\mathrm{~A})$} & \multicolumn{2}{c|}{$F_{t h}(\mathrm{mN})$} & \multicolumn{2}{|c|}{$\eta_{a}$} \\
\cline { 5 - 12 } & & & & HPHall & VF-5 & HPHall & VF-5 & HPHall & VF-5 & HPHall & VF-5 \\
\hline 300.3 & 0.18 & 0.02 & 10.0 & 9.85 & 9.96 & 7.79 & 7.38 & 182 & 186 & $54.8 \%$ & $56.6 \%$ \\
400.8 & 0.20 & 0.018 & 10.0 & 7.95 & 8.00 & 6.34 & 6.40 & 171 & 173 & $55.3 \%$ & $56.3 \%$ \\
500.0 & 0.09 & 0.02 & 10.0 & 6.94 & 6.97 & 5.48 & 5.58 & 165 & 169 & $54.8 \%$ & $57.5 \%$ \\
\hline
\end{tabular}

The $300 \mathrm{~V}$ condition displays some odd behavior, with the predicted anode efficiency being lower in spite of the higher ion current. This indicates that a decrease in some partial efficiency is outweighing the increase in current utilization. Similar agreement to that at $400 \mathrm{~V}$ has been achieved when applying HPHall to other thrusters at 300 and $400 \mathrm{~V},{ }^{8,9}$ suggesting that better agreement for $300 \mathrm{~V}$ operation can be achieved simply by tuning the electron mobility model further. However, the present level of agreement is considered adequate for determining the model's accuracy at higher voltages. The $500 \mathrm{~V}$ operating point differs most from the conditions modeled using HPHall in the past, but the code is still able to predict the thruster performance at that condition to within $3 \%$ for discharge current, ion current, and thrust. This suggests that any changes in the plasma between 400 and $500 \mathrm{~V}$ operation are not so significant as to affect the simulated thruster performance. 


\section{B. Time-averaged plasma properties}

The purpose of this section is to evaluate the ability of the model to predict internal plasma properties of the HiVHAc EDU2 at $500 \mathrm{~V}$. At this time, measurements of the HiVHAc's internal plasma properties have not yet been performed, but the accuracy of HPHall in predicting time-averaged plasma properties at low discharge voltages is well established. ${ }^{8,9}$ Hence, the predicted plasma properties at $500 \mathrm{~V}$ operation will be compared to those at 300 and $400 \mathrm{~V}$ in order to evaluate any changes that may occur at high discharge voltages.

Figure 1 shows the time-averaged plasma potential and electron temperature along the thruster centerline for $300 \mathrm{~V}$ operation. The thruster exit plane corresponds to an axial position of zero. The peak electron temperature occurs at approximately the same location as the peak electric field, as expected. The peak value of the electron temperature is about $22 \mathrm{eV}$. Upstream of the thruster exit plane the plasma potential and electron temperature be-

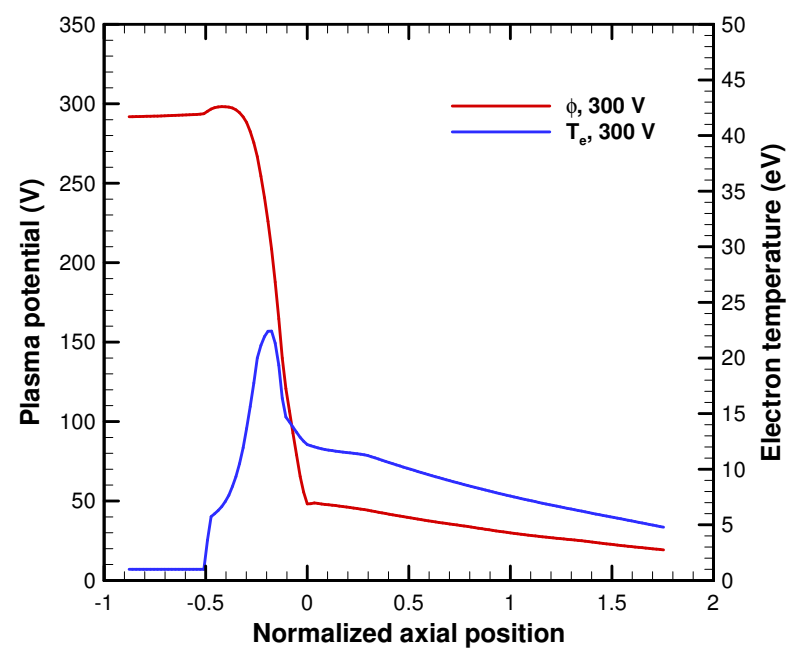

Figure 1: Plasma potential and electron temperature along the thruster centerline for $300 \mathrm{~V}$ operation. have as expected. The potential increases by a few volts from the anode potential, then falls rapidly down to about $50 \mathrm{~V}$. The small increase in the plasma potential upstream of the acceleration zone may be caused by an electron mobility near the anode that is too high compared to that in the acceleration zone, resulting in an electric field pointing toward the anode. Adjustment of the electron diffusion coefficients will likely reduce or eliminate the "bump" in the potential. The electron temperature is low near the anode, then rapidly rises to its peak in the acceleration region before decreasing again in the plume. Downstream of the exit plane, there are small gradients in the plasma potential and electron temperature that are not observed experimentally, but are typical of HPHall simulations using the three-region mobility model. ${ }^{8}$ Overall, the centerline values are qualitatively consistent with what has been predicted by HPHall for other thrusters and should serve as a reasonable point of comparison for the $500 \mathrm{~V}$ case.

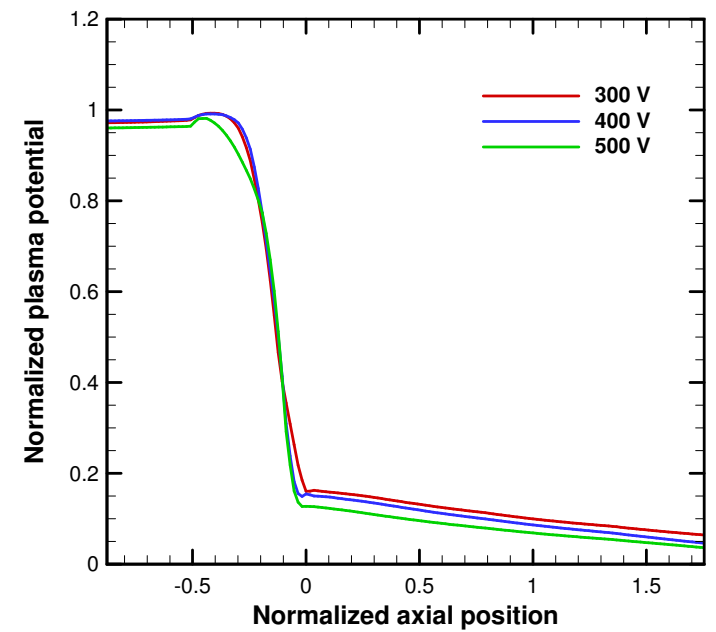

(a)

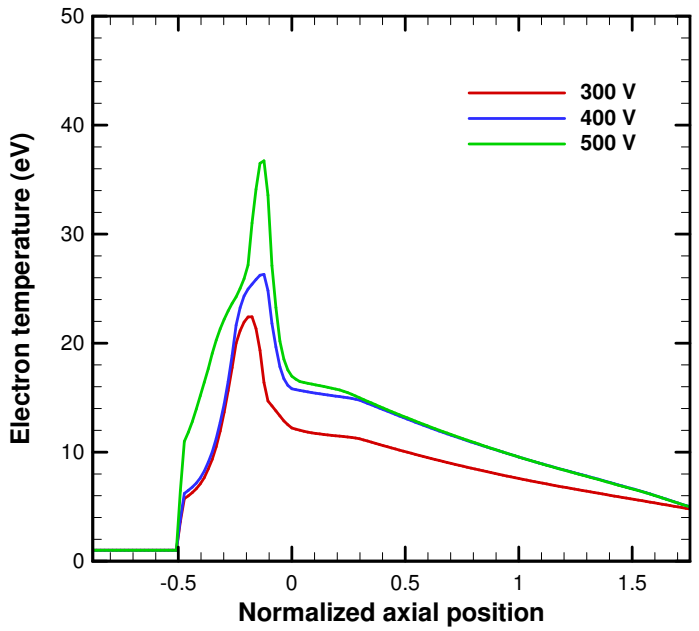

(b)

Figure 2: (a) Normalized plasma potential and (b) electron temperature profiles along the discharge channel centerline. 
Figure 2a shows plasma potential profiles normalized by the nominal discharge voltage for each of the three operating points simulated. The 300 and $400 \mathrm{~V}$ cases agree very well with one another, with the peak electric field appearing in the same location and a similar potential gradient appearing in the plume. The two cases also predict the same bump in the plasma potential just upstream of the acceleration zone. The $500 \mathrm{~V}$ case, on the other hand, differs slightly from the other two. The bump in the potential appears to occur deeper in the channel, and the potential starts to fall deeper in the channel, as well, suggesting that the acceleration zone has recessed slightly compared to the lower-voltage cases. In addition, the normalized potential at the exit plane seems to decrease slightly with increasing discharge voltage, though this may only mean that the non-normalized potential at the exit plane is quite similar between cases. Overall, the differences are quite small, on the order of a few percent, and the bulk of the acceleration zone appears in approximately the same location for all three cases. Hence, it appears that HPHall is predicting the plasma potential profile to an acceptable degree of accuracy even at high discharge voltages.

Figure $2 \mathrm{~b}$ shows the electron temperature profiles for each operating point. These profiles show the expected trend of increasing electron temperature with increasing discharge voltage. The electron temperature peaks at $22 \mathrm{eV}$ at $300 \mathrm{~V}$ nominal operation, at $27 \mathrm{eV}$ at $400 \mathrm{~V}$, and at $37 \mathrm{eV}$ at $500 \mathrm{~V}$. In terms of Hall thruster operation, increasing electron temperature usually means more multiply-charged ion species, which in turn means a reduction in the charge and mass utilization efficiencies of the thruster. The predicted performance in Section III-A, however, seems to suggest that these effects are too small to result in a significant difference between the simulations and the experiments. The addition of triply-charged ions to the model, for example, should cause the predicted thruster efficiency to decrease, resulting in worse agreement between the model and the measurements. Furthermore, the current fraction carried by doubles in the simulation increases by only a few percent at high discharge voltage, going from $17 \%$ at $300 \mathrm{~V}$ to $19 \%$ at $500 \mathrm{~V}$. If triples were included in the simulations, then it could be assumed that their current fraction would be a few percent at most. It is possible that at even higher discharge voltages the effects of multiply-charged species will become important, but for the throttling points modeled in this work that does not appear to be the case.

Figure 3 compares contours of axial ion current density at each simulated operating condition. For all three cases, there is a small region of negative current density just upstream of the bulk of the ion beam. This is most likely a result of ions generated in the region upstream of the acceleration zone where the electric field points towards the anode. As stated previously, adjustment of the mobility model is expected to correct this behavior. At higher mass flow rates, the expected increase in overall current density is observed. There appears to be a slight reduction in the beam divergence with increasing discharge voltage, but the decrease in total beam current makes a purely qualitative comparison difficult. To quantify this effect, one can compute

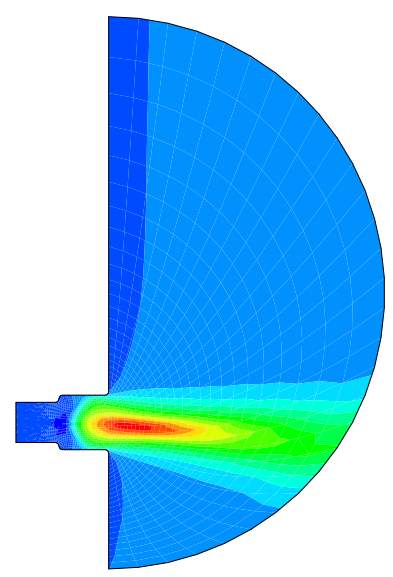

(a)

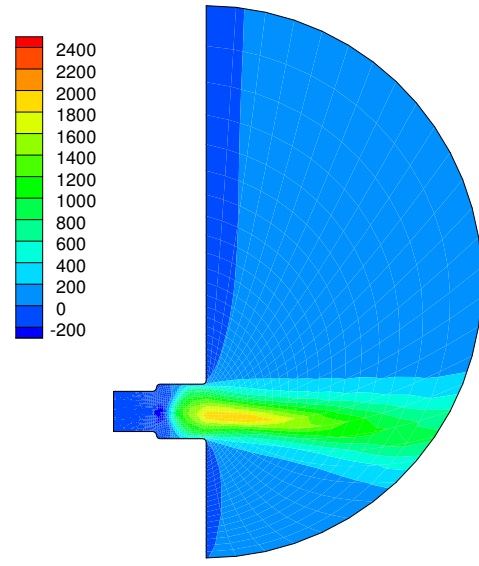

(b)
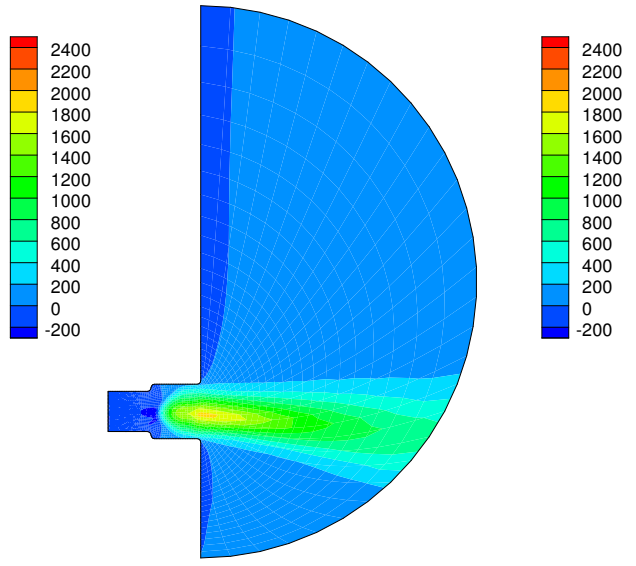

(c)

Figure 3: Comparison of axial ion current density $\left(A / \mathrm{m}^{2}\right)$ contours for (a) 300 , (b) 400, and (c) $500 \mathrm{~V}$ operation. 


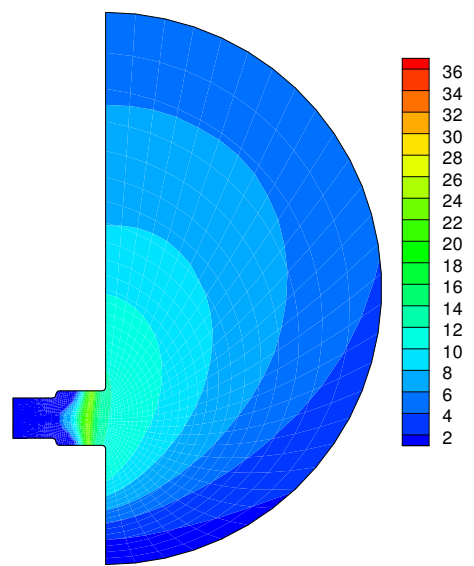

(a)

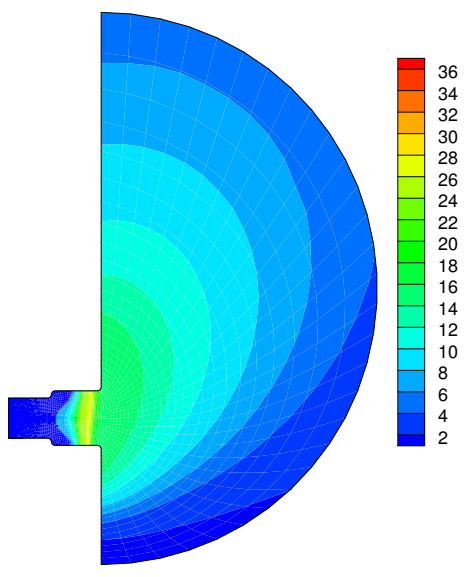

(b)

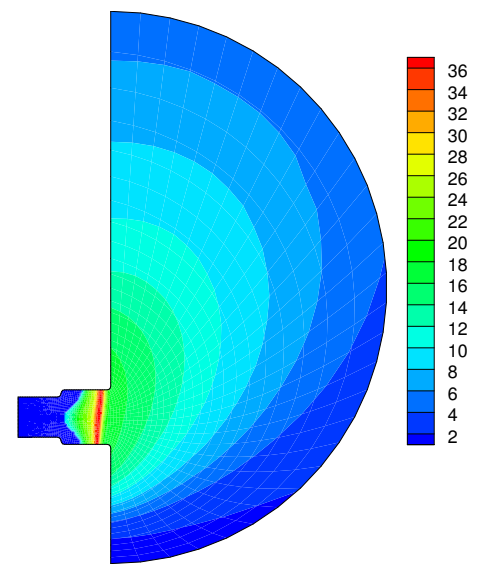

(c)

Figure 4: Comparison of electron temperature (eV) contours for (a) 300, (b) 400, and (c) $500 \mathrm{~V}$ operation.

the charge-weighted divergence angle of the beam: ${ }^{15}$

$$
\cos \delta_{j}=\frac{2 \pi \int_{r_{1}}^{r_{2}} j_{z}(r) \cos (\theta) r \mathrm{~d} r}{2 \pi \int_{r_{1}}^{r_{2}} j_{z}(r) r \mathrm{~d} r}, \cos (\theta)=\frac{z}{\sqrt{r^{2}+z^{2}}} .
$$

Equation (5) would typically be used to reduce radially-swept Faraday probe data wherein the axial location $z$ of the probe is several thruster diameters downstream so that the thruster can be treated as point source. The numerical domain used in this study is not large enough to allow data to be collected so far downstream of the thruster, but a charge-weighted divergence angle computed nearer to the thruster may be adequate for making comparisons between the three operating points.

The charge-weighted divergence of the beam is computed according to Equation (5) for each case at an axial location of about 1.4 channel lengths downstream of the thruster exit. The radial extent of the integration is about 3 channel lengths. The computed divergence angles are $46.9^{\circ}$ at $300 \mathrm{~V}, 47.1^{\circ}$ at $400 \mathrm{~V}$, and $46.8^{\circ}$ at $500 \mathrm{~V}$. All of these values appear quite high, but that may simply be a consequence of the axial location of the computation being very close to the thruster. The divergence angles also do not differ greatly from one another, suggesting either that HPHall predicts little change in beam divergence with discharge voltage or that the computation is performed too close to the thruster to reveal any such change. Ultimately, no firm conclusions can be drawn as to the divergence of the ion beam with increasing discharge voltage in these simulations without modeling the plume farther downstream of the thruster.

Shown in Figure 4 are contours of electron temperature for each of the modeled operating points. As with the centerline profiles, the electron temperature appears to be strongly correlated with discharge voltage. The region of peak electron temperature, which corresponds to the acceleration zone, is recessed into the channel by about $10-15 \%$ of the total channel length. The location of the acceleration zone is critical in determining the channel erosion, and is determined primarily by the cross-field mobility of the electrons. In the absence of internal measurements, however, it is difficult to determine whether the location of the acceleration zone in the simulation is correct. For the time being, one can only conclude that HPHall best predicts HiVHAc's performance when the acceleration zone is recessed into the discharge channel. The HiVHAc team at GRC is exploring an effort to characterize HiVHAc's internal plasma using wall-mounted probes, which would make further validation of the model possible.

\section{Discharge current oscillations}

Hall thruster discharge oscillations occur on a much shorter time scale than the channel wall erosion, but play a critical role in determining the thruster performance and internal plasma properties. Many different oscillation modes are known to exist in Hall thrusters, covering a wide range of frequencies. ${ }^{16}$ Some of these 


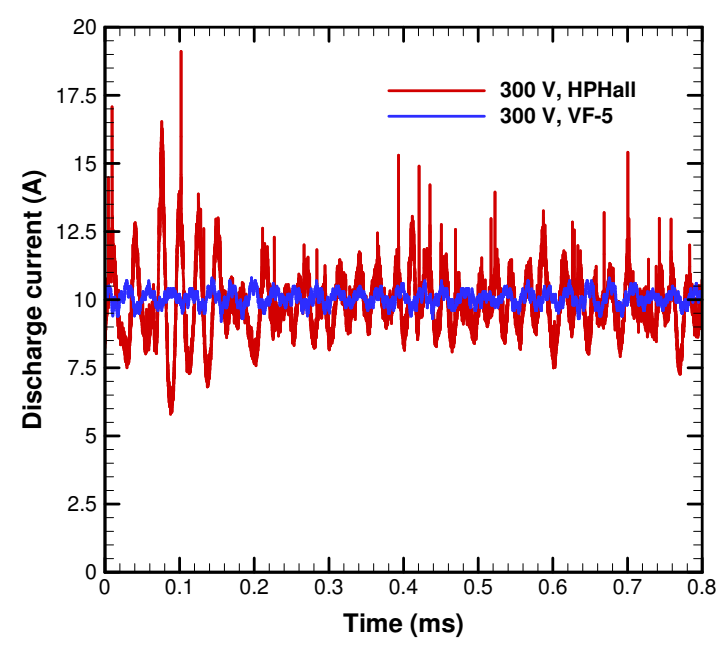

(a)

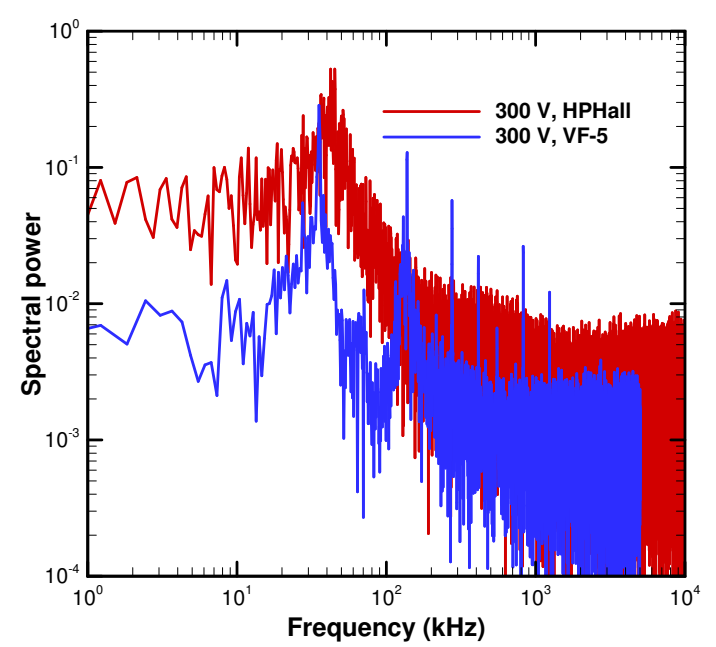

(b)

Figure 5: (a) Discharge current as a function of time and (b) power spectra of the simulated and measured discharge current oscillations at $300 \mathrm{~V}$ operation.

modes are axial in nature, but many are azimuthal. Since HPHall assumes axisymmetry, it is incapable of capturing azimuthal perturbations, but has been shown to predict axial oscillations very well in the past. ${ }^{1,16-18}$ Thus, in evaluating HPHall's ability to model high-voltage thrusters, a comparison of the oscillation modes predicted in HPHall against those measured experimentally is extremely valuable.

Figure 5a shows the computed and measured discharge current waveforms at the $300 \mathrm{~V}$ operating point and Figure 5b shows the corresponding power spectra. The first difference worth noting is that the model predicts discharge oscillations that are much greater in amplitude than those measured experimentally. The peak-to-peak amplitude of the simulated waveform is about $30 \%$ of the mean discharge current on average, whereas the peak-to-peak amplitude of the measured waveform is only $10 \%$ of the mean. The power spectra agree somewhat better, with peak frequencies of $42 \mathrm{kHz}$ and $35 \mathrm{kHz}$ for the simulated and measured waveforms, respectively, but the measured waveform also displays power spikes at $140 \mathrm{kHz}$ and its harmonics. These higher-frequency oscillations are not considered to result from physical processes in the plasma and are discussed further below.

The simulated oscillations appear to be consistent with the large-amplitude, low-frequency breathing mode oscillations commonly observed in Hall thrusters. ${ }^{1,16,17,19}$ Such axial oscillations typically occur at a frequency of $10-25 \mathrm{kHz}$, but one-dimensional predator-prey models of the Hall thruster discharge suggest that the frequency of the breathing mode scales inversely with the length of the acceleration zone. ${ }^{17}$ Hence, a high frequency is merely an indication that the ion acceleration occurs over a short distance. The origin of the experimentally observed oscillations is less clear. It is possible that they are also associated with the breathing mode. If that is the case, then there may be a damping mechanism present in the real thruster that is not included in the model. Given the sensitivity of discharge oscillations in HPHall to numerical parameters, ${ }^{20}$ it is also possible that further adjustment of the code's electron submodel will reduce the amplitude of the predicted oscillations, resulting in better agreement with those seen in the experiment. Yet another possibility is that the oscillations observed in the real thruster are not axial breathing mode oscillations, but rather azimuthal "spoke" mode oscillations. ${ }^{16}$ If that is true, then HPHall cannot capture the observed oscillations because it assumes the flow is axisymmetric.

Figures 6 and 7 show the discharge current waveforms and associated power spectra for 400 and $500 \mathrm{~V}$ operation, respectively. As at $300 \mathrm{~V}$, the amplitude of the simulated oscillations is much greater than that measured experimentally, suggesting that the difference in amplitude is not associated with changes in discharge voltage. The power spectra of the simulated waveforms indicate dominant frequencies of 41 and $48 \mathrm{kHz}$ at 400 and $500 \mathrm{~V}$, respectively. The spectra of the measured waveforms show low-frequency peaks of 35 and $25 \mathrm{kHz}$ at 400 and $500 \mathrm{~V}$ and high-frequency peaks at about $140 \mathrm{kHz}$ and its harmonics for both waveforms. The consistent appearance of the $140 \mathrm{kHz}$ peak at all operating points suggests that this frequency 


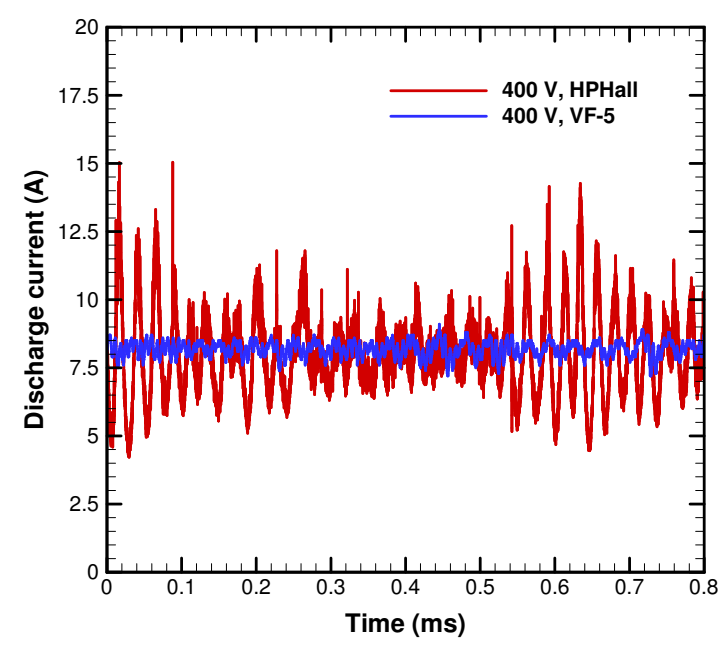

(a)

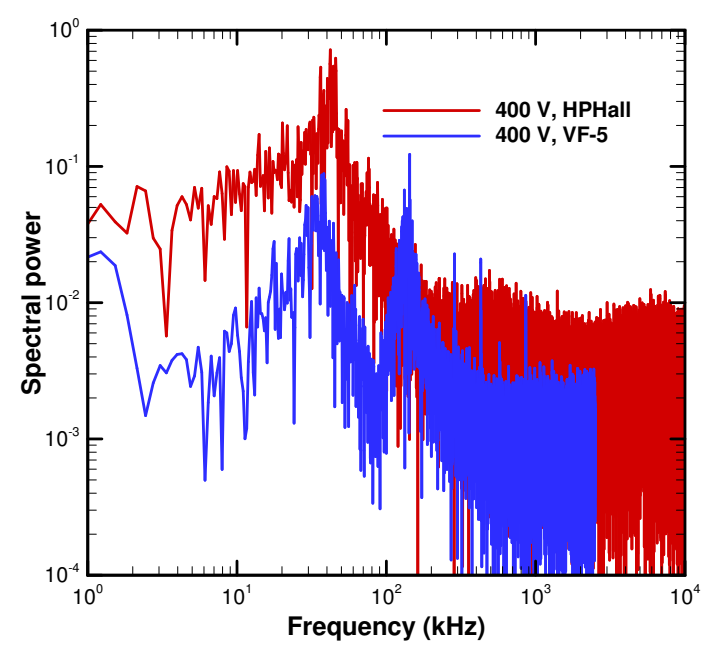

(b)

Figure 6: (a) Discharge current as a function of time and (b) power spectra of the simulated and measured discharge current oscillations at $400 \mathrm{~V}$ operation.

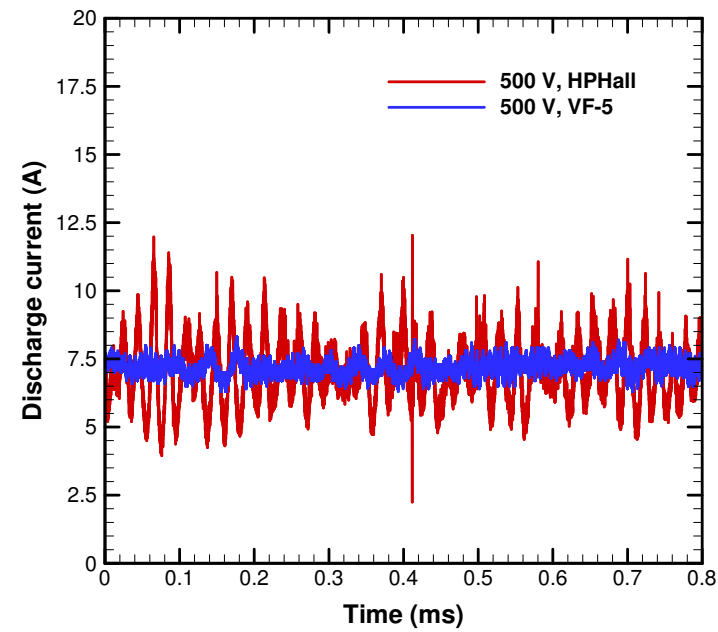

(a)

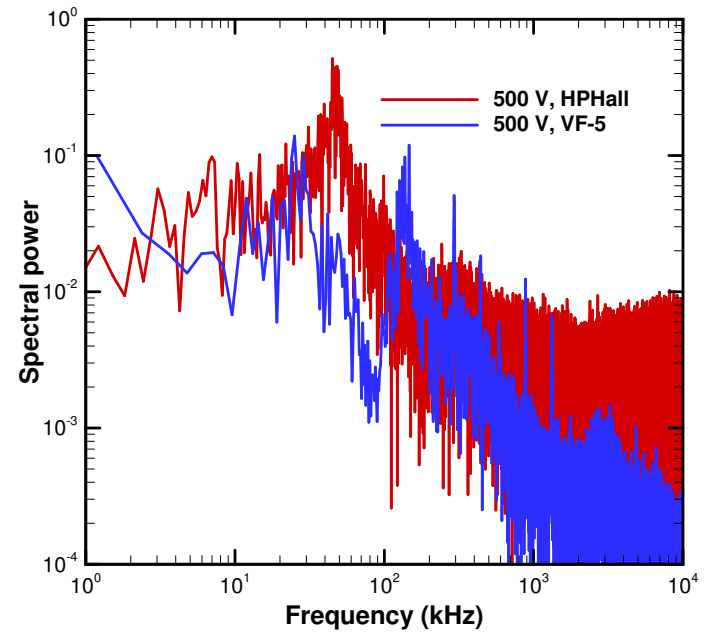

(b)

Figure 7: (a) Discharge current as a function of time and (b) power spectra of the simulated and measured discharge current oscillations at $500 \mathrm{~V}$ operation.

and its harmonics are a result of interference from the environment or connected test equipment. Hence, only the lower-frequency modes are considered to result from physical processes in the thruster plasma.

As is the case for $300 \mathrm{~V}$ operation, the discharge oscillations predicted by HPHall at 400 and $500 \mathrm{~V}$ appear to be high-amplitude breathing mode oscillations. The origin of the oscillations present in the experimental data is still unclear. At $400 \mathrm{~V}$, the predicted and measured waveforms show very similar characteristic frequencies of $38 \mathrm{kHz}$ and $35 \mathrm{kHz}$, respectively. At $500 \mathrm{~V}$, however, the predicted oscillations occur at $48 \mathrm{kHz}$, approximately twice the frequency of the measured oscillations. Curiously, the frequency of the measured oscillations appears to decrease with increasing discharge voltage. A detailed, time-resolved investigation of the breathing mode in a cluster of $600 \mathrm{~W}$ Hall thrusters indicates that the breathing mode frequency of a Hall thruster should increase with increasing discharge voltage. ${ }^{21}$ This trend is also predicted by the 1D predator-prey model of the breathing mode. ${ }^{16,17}$ The fact that the characteristic frequency of the discharge 
oscillations present in HiVHAc appears to decrease, contrary to theoretical predictions and experimental observation of other thrusters, seems to suggest that these oscillations are not related to the breathing mode. Without further experimental evidence, however, no firm conclusion regarding the origin of those oscillations can be made. GRC's HiVHAc team plans to perform high-speed camera imaging of HiVHAc EDU2 in the future, which will allow stronger qualitative and quantitative comparisons of the time-resolved thruster behavior between the simulations and experiments. For the time being, it can only be concluded that the large difference in amplitude between the simulated and measured discharge current oscillations is not sensitive to the discharge voltage.

\section{Conclusions and future work}

In this work, the ability of the hybrid-PIC model HPHall to accurately predict the performance of HiVHAc, a high-voltage, high- $I_{s p}$ Hall thruster, has been demonstrated at discharge voltages up to $500 \mathrm{~V}$. At $500 \mathrm{~V}$ operation, the computed discharge current, ion current, and thrust differ from measured values by no more than $3 \%$. Comparison of computed plasma potential and electron temperature contours at $500 \mathrm{~V}$ to those at 300 and $400 \mathrm{~V}$ suggest that the model is capable of predicting the approximate location of the thruster's acceleration zone. The expected increase in electron temperature with increasing discharge voltage is observed, along with a small increase in the current fraction carried by doubly-charged ions. An analysis of simulated and measured discharge current waveforms shows that the model predicts discharge current oscillations that are much greater in amplitude than those measured experimentally. Since these differences occur over the entire range of discharge voltages tested in this work, it is concluded that the differences are not strongly dependent on discharge voltage. A frequency domain analysis suggests that the observed oscillations may not be related to the axial breathing mode predicted by HPHall, but without further experimental data no firm conclusions can be drawn. Overall, HPHall demonstrates an ability to accurately predict thruster performance at discharge voltages up to $500 \mathrm{~V}$ without modification to the code and appears to predict the expected changes in time-averaged plasma properties with increasing discharge voltage.

Since HiVHAc is designed to run at discharge voltages up to $650 \mathrm{~V}$, validating HPHall at such discharge voltages will be necessary before it can be used as a tool for predicting channel erosion rates in the thruster. The characteristics of the plasma discharge may change significantly at such high voltages, so extension of the model must be considered in order to account for new phenomena at those conditions. The presence of additional ion species, in particular, may be worth investigating. As the model becomes better validated at high discharge voltages, it will be coupled to a database of sputter yields and velocity distribution functions generated by a high-fidelity, high-speed molecular dynamics model of the sputtering of boron nitride by xenon ions. The model will then be used to predict the erosion of the discharge channel walls and the transport of the erosion products through the thruster discharge.

\section{Acknowledgments}

The research described in this paper was supported with the funding of a NASA Space Technology Research Fellowship, grant \#NNX11AM64H.

\section{References}

${ }^{1}$ Fife, J., Hybrid-PIC Modeling and Electrostatic Probe Survey of Hall Thrusters, Ph.D. thesis, Massachusetts Institute of Technology, 1999.

${ }^{2}$ Gamero-Castaño, M. and Katz, I., "Estimation of Hall Thruster Erosion Using HPHall," 29th International Electric Propulsion Conference, No. 2005-303, Princeton, New Jersey, USA, Oct. 2005.

${ }^{3}$ Parra, F. I., Ahedo, E., Fife, J. M., and Martinez-Sanchez, M., "A two-dimensional hybrid model of the Hall thruster discharge," Journal of Applied Physics, Vol. 100, No. 2, 2006.

${ }^{4}$ Parra, F., Escobar, D., and Ahedo, E., "Improvements on particle accuracy in a Hall thruster hybrid code," 42nd AIAA/ASME/SAE/ASEE Joint Propulsion Conference \&S Exhibit, No. 2006-4830, Sacramento, California, USA, July 2006.

${ }^{5}$ Escobar, D. and Ahedo, E., "Improved electron formulation for a Hall thruster hybrid model," 42nd AIAA/ASME/SAE/ASEE Joint Propulsion Conference \& Exhibit, No. 2006-4326, Sacramento, California, USA, July 2006.

${ }^{6}$ Hofer, R. R., Katz, I., Mikellides, I., and Gamero-Castaño, M., "Heavy Particle Velocity and Electron Mobility Modeling in Hybrid-PIC Hall Thruster Simulations," 42nd AIAA/ASME/SAE/ASEE Joint Propulsion Conference E Exhibit, No. 20064658, Sacramento, California, USA, July 2006. 
${ }^{7}$ Hofer, R. R., Mikellides, I., Katz, I., and Goebel, D., "Wall sheath and electron mobility modeling in hybrid-PIC Hall thruster simulations," 43rd AIAA/ASME/SAE/ASEE Joint Propulsion Conference \& Exhibit, No. 2007-5267, Cincinnati, Ohio, USA, 2007.

${ }^{8}$ Hofer, R. R., Katz, I., Mikellides, I., Goebel, D. M., Jameson, K. K., Sullivan, R. M., and Johnson, L. K., "Efficacy of Electron Mobility Models in Hybrid-PIC Hall Thruster Simulations," 44th AIAA/ASME/SAE/ASEE Joint Propulsion Conference \&S Exhibit, No. 2008-4924, Hartford, Connecticut, USA, July 2008.

${ }^{9}$ Hofer, R. R., Mikellides, I. G., Katz, I., and Goebel, D. M., "BPT-4000 Hall thruster discharge chamber erosion model comparison with qualification life test data," 30th International Electric Propulsion Conference, Florence, Italy, 2007.

${ }^{10}$ Cheng, S., Modeling of Hall thruster lifetime and erosion mechanisms, Ph.D. thesis, Massachusetts Institute of Technology, 2007.

${ }^{11}$ Kamhawi, H., Manzella, D., Pinero, L., Haag, T., Mathers, A., and Liles, H., "In-Space Propulsion High Voltage Hall Accelerator Development Project Overview," 45th AIAA/ASME/SAE/ASEE Joint Propulsion Conference \& Exhibit, No. 2009-5282, Denver, Colorado, USA, Aug. 2009.

${ }^{12}$ Kamhawi, H., Manzella, D., Pinero, L., Haag, T., and Huang, W., "In-Space Propulsion High Voltage Hall Accelerator Development Project Overview," 46th AIAA/ASME/SAE/ASEE Joint Propulsion Conference E3 Exhibit, No. 2010-6860, Nashville, Tennessee, USA, July 2010.

${ }^{13}$ Kamhawi, H., Haag, T., Huang, W., Shastry, R., Pinero, L., Peterson, T., and Mathers, A., "Performance and Environmental Test Results of the High Voltage Hall Accelerator Engineering Development Unit," 48th AIAA/ASME/SAE/ASEE Joint Propulsion Conference \&S Exhibit, No. 2012-3854, Atlanta, Georgia, USA, July 2012.

${ }^{14}$ Huismann, T., Improving Hall Thruster Plume Simulation through Refined Characterization of Near-field Plasma Properties, Ph.D. thesis, University of Michigan, 2011.

${ }^{15}$ Huang, W., Kamhawi, H., and Shastry, R., 48th AIAA/ASME/SAE/ASEE Joint Propulsion Conference 83 Exhibit, No. 2012, Atlanta, Georgia, USA, July.

${ }^{16}$ Choueiri, E. Y., "Plasma oscillations in Hall thrusters," Physics of Plasmas, Vol. 8, No. 4, April 2001, pp. 1411.

${ }^{17}$ Fife, J. M., Martinez-Sanchez, M., and Szabo, J., "A numerical study of low-frequency discharge oscillations in Hall thrusters," 33rd AIAA/ASME/SAE/ASEE Joint Propulsion Conference \& Exhibit, Seattle, Washington, USA, July 1997.

${ }^{18}$ Giuliano, P. and Boyd, I., "Analysis of a plasma test cell including non-neutrality and complex collision mechanisms," 48th AIAA/ASME/SAE/ASEE Joint Propulsion Conference 86 Exhibit, Atlanta, Georgia, USA, 2012.

${ }^{19}$ Boeuf, J. and Garrigues, L., "Low frequency oscillations in a stationary plasma thruster," Journal of Applied Physics, Vol. 84, No. 7, 1998, pp. 3541.

${ }^{20}$ Giuliano, P. N. and Boyd, I. D., "Spectral analysis of simulated Hall thruster discharge current oscillations," 31st International Electric Propulsion Conference, Ann Arbor, Michigan, USA, Sept. 2009.

${ }^{21}$ Lobbia, R. B., A Time-resolved Investigation of the Hall Thruster Breathing Mode, Ph.D. thesis, University of Michigan, 2010 . 